\title{
AUTOMATED MOUNTING BIAS CALIBRATION for AIRBORNE LIDAR SYSTEM
}

\author{
Jing Zhang ${ }^{\text {a }}$, Wanshou Jiang ${ }^{\text {a, }}$, San Jiang ${ }^{\text {a }}$ \\ a LIESMARS, Wuhan University, 129 Luoyu Road, Wuhan, China \\ zhangjingwh@gmail.com, jws@Imars.whu.edu.cn, jiangsan870211@163.com
}

Commission IV, WG IV/4

KEYWORDS: LIDAR, calibration, mounting bias, bore-sight angle, lever-arm offset, Virtual Corresponding Point Model

\begin{abstract}
:
Mounting bias is the major error source of Airborne LIDAR system. In this paper, an automated calibration method for estimating LIDAR system mounting parameters is introduced. LIDAR direct geo-referencing model is used to calculate systematic errors. Due to LIDAR footprints discretely sampled, the real corresponding laser points are hardly existence among different strips. The traditional corresponding point methodology does not seem to apply to LIDAR strip registration. We proposed a Virtual Corresponding Point Model to resolve the corresponding problem among discrete laser points. Each VCPM contains a corresponding point and three real laser footprints. Two rules are defined to calculate tie point coordinate from real laser footprints. The Scale Invariant Feature Transform (SIFT) is used to extract corresponding points in LIDAR strips, and the automatic flow of LIDAR system calibration based on VCPM is detailed described. The practical examples illustrate the feasibility and effectiveness of the proposed calibration method.
\end{abstract}

\section{INTRODUCTION}

Airborne LIDAR is a very effective and accurate technology for 3D information collection, and it is widely adopted for land mapping, resource exploration, and disaster monitoring, 3D urban reconstruction and so on. A typical LIDAR system consists of three main components, a differential Global Position System (DGPS) to provide position information, an Inertial Measurement Unit (IMU) for attitude determination, and a laser scanning system to provide range and angle information (Baltsavias, 1999). As a multi-sensor integrated system, the positioning accuracy of individual laser footprint is affected by many factors. The main limiting factors contain the errors of the platform position and orientation derived from the DGPS/INS data and assembly errors related to misalign among sensors. The research of LIDAR data adjustment can eliminate systematic errors effectively and improve positioning accuracy. It is very important for LIDAR applications. Nowadays, existing methods can be divided into two categories: one is data-driven approach (often referred to as strip adjustment), the other is model-driven approach (often referred to as system calibration) (Shan, 2008).

LIDAR strip adjustment methods have been developed in the past few years. For the sake of mitigating the impact of systematic discrepancies between overlapping strips, various mathematic models are employed to approximately express LIDAR strip deformation (Kilian, 1996; Crombaghs, 2000; Maas, 2002; Vosselman, 2004; Filin, 2004). As the LIDAR system errors cause complicated discrepancies between strips, it is impossible to find an optimal generalized model satisfied all sorts of strip deformation. Actually, the model selection is mostly empirical. The adjustment precision depends on the appropriateness of model selection.

Alternatively, system calibration methods use rigorous physical model to calibrate LIDAR systematic biases. Then discrepancies between LIDAR overlapping strips can be reduced by compensating systematic errors directly. Schenk
(2001) analyzed the LIDAR systematic errors, and introduced a manual calibration method to solve each parameter individually. Due to correlation of systematic errors, most methods adopt partial parameters for calibration. For example, Morin (2002) solves the bore-sighting angles and the scanner torsion simultaneously. Skaloud (2006) adopts bore-sighting angles and laser range bias. Habib (2007) selects bore-sighting angles and lever-arm components. Compared with strip adjustment, system calibration utilized laser point raw measurements (such as ranges, mirror angles, position and orientation information for each pulse) and solved system biases directly. It is more reliable and stable.

LIDAR adjustment process relies on the identification of corresponding primitives for overlapping LIDAR strips. There are three types of corresponding primitives: point primitive, linear primitive and planar primitive. Considering discrete distribution of laser footprint, identifying corresponding point among LIDAR strips is almost impossible. Currently, most methods choose linear and planar primitives (Skaloud, 2006; Filin, 2006; Habib, 2007), and determine corresponding lines and planars manually. To ensure the accurate calibration results, these methods require lines in different directions, planars of different slope and aspect (Habib, 2010), which are difficult available in practice.

In this paper, a full automatic method of LIDAR system bias calibration is described. The rigorous model of LIDAR direct geo-referencing is used to solve bore-sighting angles and lever-arm offsets. Point primitive is used as corresponding. As real conjugated point does not exist in LIDAR strips, the Virtual Corresponding Point Model (VCPM) is proposed. Tow rules are defined for VCPM to connect virtual conjugated point with real laser footprint. Then strict correspondence between discrete footprints can be determined by VCPM. Because VCPM resolve the corresponding problem of discrete points, many sophisticated point matching method in traditional photogrammetry can be applied in LIDAR data adjustment. In the following, detailed workflow of VCPM extraction and LIDAR system calibration is introduced in chapter 2 . In chapter 
3, real dataset from Anyang, China is used to test our method. The results show the feasibility and efficiency of the proposed method.

\section{METHOD}

\subsection{Direct Geo-referencing Model}

According to LIDAR position equation (Baltsavias, 1999; Schenk, 2001), space coordinate of each laser footprint is calculated from system raw measurements. In various kinds of systemic errors, we choose bore-sighting angles and lever-arm offsets (which are known as the mounting bias) as system calibration parameters. Bore-sight angles describe the rotate angles between IMU body frame to laser scanner frame, and lever-arm offsets are position shift between GPS antenna center to laser scanner center. Compared with other systematic error sources, mounting bias is easy to change. However, each time the LIDAR system is installed, re-installed, or moved within the aircraft, small changes in the alignment may occur. So LIDAR mounting biases should be calibrated frequently to obtain high quality laser scanner data (Morin, 2002; Habib, 2008).

Up to now, differential GPS technique has demonstrated positioning accuracy at the $3-5 \mathrm{~cm}$ level. Considering only mounting bias, LIDAR position equation can be simplified as equation(1):

$$
\left[\begin{array}{c}
X \\
Y \\
Z
\end{array}\right]=\left[\begin{array}{c}
X_{g p s} \\
Y_{g p s} \\
Z_{g p s}
\end{array}\right]+\boldsymbol{R}_{i m u} \cdot\left(\boldsymbol{R}_{\text {misalign }} \cdot \boldsymbol{R}_{\text {scan }} \cdot\left[\begin{array}{c}
0 \\
0 \\
\rho
\end{array}\right]+\left[\begin{array}{c}
X_{\text {offset }} \\
Y_{\text {offset }} \\
Z_{\text {offset }}
\end{array}\right]\right)
$$

Where, $[X, Y, Z]^{T}$ is coordinates of laser footprints in the reference frame; $\left[X_{g p s}, Y_{g p s}, Z_{g p s}\right]^{T}$ is coordinates of GPS antenna center; $\boldsymbol{R}_{\text {imu }}$ is the orientation matrix from IMU body frame to reference frame parameterized by roll $(r)$, pitch $(p)$ and heading $(h)$ angle observations measured by IMU unit; $\boldsymbol{R}_{\text {misalign }}$ is defined by bore-sight angles, which designates the rotation matrix between IMU body frame and laser scanner frame. The bore-sight angles is parameterized by $[\alpha, \beta, \gamma]$; $R_{\text {scan }}$ is the rotation matrix defined by laser scanner rotation angle $\theta ; \rho$ is range measurement from laser range-finder; $\left[X_{\text {offset }}, Y_{\text {offset }}, Z_{\text {offset }}\right]^{T}$ defines the lever-arm offsets from laser scanner center to GPS antenna center.

In equation(1), we designate lever-arm offset $\left[X_{\text {offset }}, Y_{\text {offset }}, Z_{\text {offset }}\right]^{T}$ and bore-sight angles $[\alpha, \beta, \gamma]$ are unknown coefficients, all the other variants are known values which can obtain from laser point raw measurements. Observation equation of unknown coefficients is given in formula(2):

$$
\boldsymbol{L}=F(\widetilde{\boldsymbol{X}})
$$

Where, $\boldsymbol{L}=\left[X^{e}, Y^{e}, Z^{e}\right]^{T}$ is coordinate expectations of footprints; $\widetilde{\boldsymbol{X}}=\left[X_{\text {offset }}, Y_{\text {offset }}, Z_{\text {offset }}, \alpha, \beta, \gamma\right]^{T}$ is the unknown parameters of mounting parameters. Coordinate expectations can gain from ground control points, or adopt mean value of conjugate points just as self-calibration. According to formula(2), one laser point can formulate three equations. So selecting two more points, the least square solution of mounting parameters can be obtained.

Indirect adjustment model is used to solve mounting parameters, which is shown in formula(3). Where $\boldsymbol{l}=\boldsymbol{L}-\left(\boldsymbol{A} \boldsymbol{X}^{0}+\boldsymbol{\Delta}\right)=\boldsymbol{L}-\boldsymbol{L}^{0}$; $\boldsymbol{A}$ is the coefficient matrix of unknown parameters; $\boldsymbol{L}^{0}$ is observation approximation, and $\boldsymbol{X}^{0}$ is unknown parameters approximation, $\hat{\boldsymbol{x}}$ is unknown parameters correction.

$$
\boldsymbol{V}=\boldsymbol{A \hat { x }}-\boldsymbol{l}
$$

The solution is derived by least square adjustment in formula(4). $\boldsymbol{P}$ is weight matrix of observation. If all observations are of the same importance, $\boldsymbol{P}$ could be identity matrix.

$$
\hat{\boldsymbol{x}}=\left(\boldsymbol{A}^{T} \boldsymbol{P} \boldsymbol{A}\right)^{-1} \boldsymbol{A}^{T} \boldsymbol{P l}
$$

\subsection{Virtual Corresponding Point Model}

As LIDAR points are sampled discretely, real conjugated laser footprints are hardly existence. We proposed a novel method which termed Virtual Corresponding Point Model (VCPM) to resolve this corresponding problem among discrete laser points. As shown in Fig. 1, supposing point $p$ and $q$ are corresponding points in different strips. Point $p$ is not a real laser footprint, three real footprints $p 1, p 2$ and $p 3$ in its nearest neighborhood are selected to construct a triangle enclosing $p$. We integrate $p$ and $p 1, p 2, p 3$ into VCPM P. In the same way, VCPM Q can be constructed for $q$. Now two rules are defined for VCPM to calculate the horizontal and vertical coordinates of corresponding points by three laser footprints.

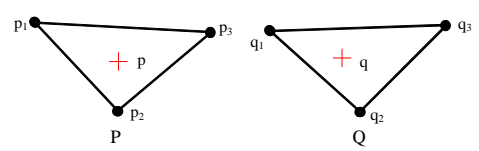

Fig. 1 Virtual Corresponding Point Model

Rule 1: Coplanar constraint

When $p$ and three relevant footprints, $p 1, p 2, p 3$, are coplanar, the vertical coordinate of $\mathrm{p}$ can be determined by linear interpolation. Let coordinates of point $p 1, \quad p 2, \quad p 3$ are $\left(X_{p_{1}}, Y_{p_{1}}, Z_{p_{1}}\right),\left(X_{p_{2}}, Y_{p_{2}}, Z_{p_{2}}\right),\left(X_{p_{3}}, Y_{p_{3}}, Z_{p_{3}}\right)$ and horizontal coordinates of $p$ is $\left(X_{p}, Y_{p}\right)$. The vertical coordinate of point $p$ is determined by equation(5):

$$
\begin{aligned}
& d X_{21}=X_{p_{2}}-X_{p_{1}} \quad d Y_{21}=Y_{p_{2}}-Y_{p_{1}} \quad d Z_{21}=Z_{p_{2}}-Z_{p_{1}} \\
& d X_{31}=X_{p_{3}}-X_{p_{1}} \quad d Y_{31}=Y_{p_{3}}-Y_{p_{1}} \quad d Z_{31}=Z_{p_{3}}-Z_{p_{1}} \\
& Z=Z_{p_{1}}+ \\
& \frac{\left(X_{p_{1}}-X\right) \cdot\left(d Y_{21} \cdot d Z_{31}-d Y_{31} \cdot d Z_{21}\right)-\left(Y_{p_{1}}-Y\right)\left(d X_{21} \bullet d Z_{31}-d X_{31} \cdot d Z_{21}\right)}{d X_{21} \bullet d Y_{31}-d X_{31} \bullet d Y_{21}}
\end{aligned}
$$

Rule 2: Rigid-body assumption

According to equation(1), the modification of mounting parameters will vary footprints coordinates, furthermore affect corresponding point coordinates. Because $p$ and $p 1, p 2, p 3$ locate in a small area, when footprint coordinates varying, assuming the entity deformation of VCPM P obeys rigid-body transformation. After mounting parameters modified, coordinates of corresponding point $p$ could be recalculated by rigid transformation (Formula(6)).

$$
\left[\begin{array}{c}
X_{p}^{1} \\
Y_{p}^{1} \\
Z_{p}^{1}
\end{array}\right]=\boldsymbol{R}_{\text {rigid }}\left[\begin{array}{c}
X_{p}^{0} \\
Y_{p}^{0} \\
Z_{p}^{0}
\end{array}\right]+\left[\begin{array}{c}
\Delta x \\
\Delta y \\
\Delta z
\end{array}\right]
$$


Where $\left[X_{p}^{0}, Y_{p}^{0}, Z_{p}^{0}\right]^{T}$ is the original coordinates of $p$; $\left[X_{p}^{1}, Y_{p}^{1}, Z_{p}^{1}\right]^{T}$ is the new coordinates of $p$ after mounting parameters changed; $R_{\text {rigid }}$ is the rotation matrix and $[\Delta x, \Delta y, \Delta z]^{T}$ is offset vector of rigid transformation. The rotation matrix and offset vector could be calculated from three real footprints. Whenever mounting parameters change, coordinates of three footprints before and after varying are used to solve rigid transformation parameters. Then using previous corresponding point coordinates and transformation parameters, current coordinates of corresponding point can be determined.

Utilizing two rules above, we could recalculate and update corresponding point coordinate using real footprints when systematic parameters varying. VCPM provides an approach to solve point corresponding in discrete LIDAR points.

\subsection{Adjustment Process}

Based on VCPM, the flowchart of LIDAR mounting bias adjust is shown in Fig. 2. The details of each step are as follows:

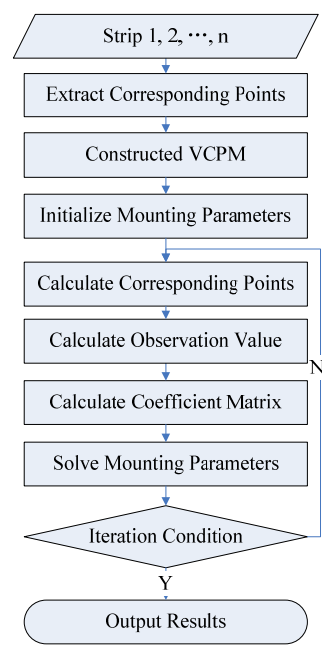

Fig. 2 Workflow of calibration

Step 1 Extract corresponding points

Corresponding points identification is the base of LIDAR system calibration. Corresponding points can be determined manually. In this paper, a full automatic approach is given. The details are introduced in chapter 2.4 .

Step 2 Construct VCPM

For each corresponding point, all real footprints within a given radius (e.g. 2 meters, depend on average point spacing) are extracted. Then, a delaunay triangular network (TIN) would be constructed from these footprints. Finally, pick up the triangle encompasses the corresponding point and take triangle vertexes into VCPM. If laser footprints within specified radius are less than 3 , TIN construction is failed. In this case, this corresponding point will be deleted.

Step 3 Initialize mounting parameters

Initialize values for all mounting parameters. These parameters can be initialized by previous calibration. In practical, mounting bias are usually very small, we can initialize all as zero.

Step 4 Calculate corresponding points coordinates

Corresponding points coordinates are calculated based on VCPM. For point $\mathrm{k}$, horizontal coordinates $\left(X_{k}, Y_{k}\right)$ are determined in extraction step. If $\mathrm{k}$ and surrounding laser footprint is coplanar, the vertical coordinate $Z_{k}$ could be interpolated according formula(5). In the iteration process of adjustment, mounting parameters will vary. Current corresponding point coordinates can be calculated by formula(6).

Step 5 Calculate Observation value

Calculate expectation value of corresponding points coordinates and constitute observation vector ( $\boldsymbol{L}$ in formula(2)). Suppose corresponding point $k$ visible in $\mathbf{n}$ strips, expectation values are calculated as(7).

$$
X_{k}^{e}=\frac{1}{n} \sum_{i=1}^{n} X_{k_{i}}
$$

Where $X_{k}^{e}$ is coordinate expectation; $X_{k_{i}}$ is coordinate in strip $i$.

Step 6Calculate Coefficient Matrix

Due to the complex relationship between observations and unknowns, it's difficult to linearize the functional model. We adopt finite difference method to calculate the coefficient matrix of unknowns. Calculation is as follows:

$$
\begin{gathered}
z=f(x, y) \\
\frac{\partial z}{\partial x}=\frac{f(x+\Delta x, y)-f(x-\Delta x, y)}{2 \Delta x} \\
\frac{\partial z}{\partial y}=\frac{f(x, y+\Delta y)-f(x, y-\Delta y)}{2 \Delta y}
\end{gathered}
$$

For equation(8), $x, y$ are unknowns, partial derivatives can be calculated in formula(9). Coefficient matrix of mounting parameters is formulated as(10):

$$
A=\left[\begin{array}{llllll}
\frac{\partial X^{e}}{\partial X_{\text {offset }}} & \frac{\partial X^{e}}{\partial Y_{\text {offset }}} & \frac{\partial X^{e}}{\partial Z_{\text {offset }}} & \frac{\partial X^{e}}{\partial \alpha} & \frac{\partial X^{e}}{\partial \beta} & \frac{\partial X^{e}}{\partial \gamma} \\
\frac{\partial Y^{e}}{\partial X_{\text {offset }}} & \frac{\partial Y^{e}}{\partial Y_{\text {offset }}} & \frac{\partial Y^{e}}{\partial Z_{\text {offset }}} & \frac{\partial Y^{e}}{\partial \alpha} & \frac{\partial Y^{e}}{\partial \beta} & \frac{\partial Y^{e}}{\partial \gamma} \\
\frac{\partial Z^{e}}{\partial X_{\text {offset }}} & \frac{\partial Z^{e}}{\partial Y_{\text {offset }}} & \frac{\partial Z^{e}}{\partial Z_{\text {offset }}} & \frac{\partial Z^{e}}{\partial \alpha} & \frac{\partial Z^{e}}{\partial \beta} & \frac{\partial Z^{e}}{\partial \gamma}
\end{array}\right]
$$

Step 7 Solve mounting parameters

Mounting parameters are solved by least square. It is a classical parameters minimization problem, among various solutions, we choose LM algorithm (MORÉ, 1978).

Step 8 Iteration Condition

Once residual error of corresponding points is less than a threshold or calibration parameters are converged, stop the iteration and output the adjustment results. Otherwise, go to Step 4.

\subsection{Automatic Corresponding Point Extraction}

VCPM provides an approach to solve point corresponding in discrete LIDAR points. So many sophisticated points matching method in traditional photogrammetry can be employed to extract corresponding points in LIDAR data. In this section, automatic corresponding point extraction is introduced in 5 steps.

(1) Interpolation

Interpolate discrete LIDAR point cloud to regular grid. Then reliable image matching methods can recommend to determine corresponding points. LIDAR data provides both elevation information and intensity information. The intensity of laser footprints represents the reflectivity characteristics of targets. It is beneficial for matching (Abedini, 2008). So we interpolate the intensity of each LIDAR strip to regular grid individually. 
(2) Matching by SIFT

SIFT features (Lowe, 2004) are extracted from each interpolated LiDAR strip. Then the features are matched between pair of strips.

(3) RANSAC Estimation

Since there might be some mismatches in SIFT matching, RANSAC estimation (Fischler, 1981) is used to select correct matches. We use affine transformation as a geometrical constraint in this step.

(4) Tracking

With a set of geometrically consistent matches between each strip pair, a tracking procedure is employed to connect corresponding points across multiple strips.

(5) Classification of corresponding points

Vertical coordinates of corresponding points are determined by linear interpolation. When corresponding point and surrounding laser footprints are not coplanar, the vertical coordinate is unreliable. Surface roughness is adopted to analysis and classify all accepted corresponding points (Shepard, 2001). Take each corresponding point as center, statistics RMSE (Root Mean Square Error) of laser footprints elevation within a specified radius (e.g. $2 \mathrm{~m}$ ), and use this value measure surface roughness. When corresponding points lie on vegetation or building edges the value of surface roughness is relatively big. When corresponding points lie on steady planes, the roughness is relatively small. So we compare the surface roughness with a given threshold $(0.25)$ to classify the corresponding points into two types: horizontal-vertical points and horizontal points. In the process of adjustment, for horizontal-vertical points, $\mathrm{x}, \mathrm{y}, \mathrm{z}$ equations are used; for horizontal points, only $\mathrm{x}, \mathrm{y}$ equations are used.

\section{EXPERIMENT AND ANALYSIS}

\subsection{Experiment Description}

To test the proposed method, real LIDAR dataset is used for experiment. Experimental area is in urban area located at Anyang, Henan province, China. The terrain is relatively flat, and plenty of buildings distribute in this area. LIDAR dataset is acquired with ALS50II. The whole dataset contains 8 LIDAR strips (shown in Fig. 3), and details of each strip is list in Tab. 1.

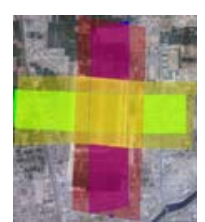

a. $1 \sim 4$ strips

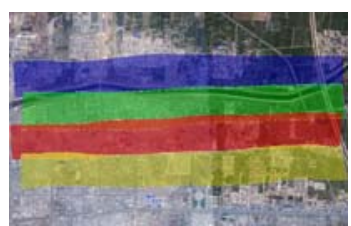

b. $5 \sim 8$ strips
Fig. 3 LIDAR strips in experimental area

\begin{tabular}{|c|c|c|c|c|}
\hline $\begin{array}{c}\text { Strip } \\
\text { ID }\end{array}$ & Direction & $\begin{array}{c}\text { Average } \\
\text { Flight Height }\end{array}$ & $\begin{array}{c}\text { Average point } \\
\text { Spacing }\end{array}$ & Colour \\
\hline 1 & W-E & $760 \mathrm{~m}$ & $0.5 \mathrm{~m}$ & Green \\
\hline 2 & S-N & $760 \mathrm{~m}$ & $0.5 \mathrm{~m}$ & Blue \\
\hline 3 & W-E & $1310 \mathrm{~m}$ & $1.0 \mathrm{~m}$ & Yellow \\
\hline 4 & N-S & $1310 \mathrm{~m}$ & $1.0 \mathrm{~m}$ & Red \\
\hline 5 & W-E & $500 \mathrm{~m}$ & $0.5 \mathrm{~m}$ & Blue \\
\hline 6 & E-W & $500 \mathrm{~m}$ & $0.5 \mathrm{~m}$ & Green \\
\hline 7 & W-E & $500 \mathrm{~m}$ & $0.5 \mathrm{~m}$ & Red \\
\hline 8 & E-W & $500 \mathrm{~m}$ & $0.5 \mathrm{~m}$ & Yellow \\
\hline
\end{tabular}

Tab. 1 Essential information of 8 strips

8 strips can be divided into two groups. Strips 1-4 are four cross strips; the overlap of the same direction strips is 100 percent. Strips 5-8 are four parallel strips; the maximal overlap between strips is 15 percent. In order to inspect experimental result, we assign a different color for each strip (Tab. 1).

The experiment is composed of two steps. Firstly, Strips 1-4 are used to confirm the feasibility of the proposed method. Then the calibration results of mounting bias are used to correct remaining 4 strips. Quantitative analysis of relative accuracy after correction is given to confirm the efficiency of the proposed method.

\subsection{Results Analysis}

3.2.1 Experiment 1: In this experiment, strips 1-4 are used to solve LIDAR system mounting parameters. Firstly, SIFT features are extracted form interpolated LIDAR intensity image. The number of original feature points of each strip is shown in. Then SIFT features are matched for each pair of strips, the initial pairwise matches are shown in Tab. 2. The value in row $i$, column $\mathrm{j}$ is the match number between image pair ( $\mathrm{i}, \mathrm{j})$. After RANSAC estimation, geometrically consistent matches are shown in Tab. 3. Finally, after tracking and classification, the number of received corresponding points is shown in Tab. 4. The distribution of corresponding points is shown in Fig. 4.

\begin{tabular}{|l|l|l|l|l|}
\hline Strip & 1 & 2 & 3 & 4 \\
\hline Count & 12813 & 17909 & 30979 & 33220 \\
\hline \multicolumn{6}{|c|}{ Tab. 2 Original SIFT feature points }
\end{tabular}

\begin{tabular}{|l|l|l|l|l|}
\hline Strip & 1 & 2 & 3 & 4 \\
\hline 1 & 0 & 31 & 717 & 90 \\
\hline 2 & - & 0 & 80 & 683 \\
\hline 3 & - & - & 0 & 245 \\
\hline 4 & - & - & - & 0 \\
\hline
\end{tabular}

Tab. 3 Initial pairwise matches

\begin{tabular}{|l|l|l|l|l|}
\hline Strip & 1 & 2 & 3 & 4 \\
\hline 1 & 0 & 13 & 289 & 36 \\
\hline 2 & - & 0 & 35 & 301 \\
\hline 3 & - & - & 0 & 89 \\
\hline 4 & - & - & - & 0 \\
\hline
\end{tabular}

Tab. 4 Matches of RANSAC estimation

\begin{tabular}{|l|l|}
\hline Horizontal - Vertical & 312 \\
\hline Horizontal & 426 \\
\hline Total Num & 738 \\
\hline
\end{tabular}

Tab. 5 Received tie points

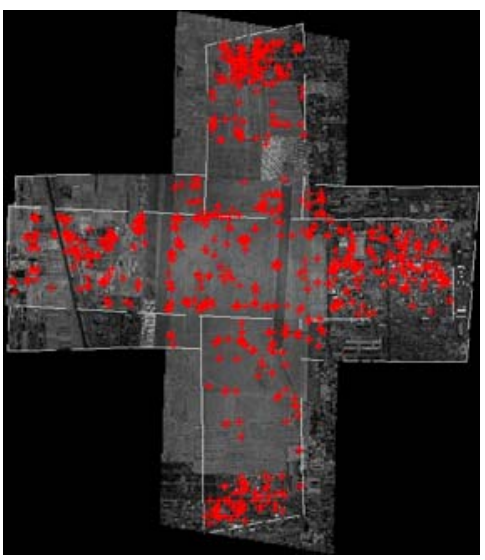

Fig. 4 Distribution of final tie points

The solved mounting parameters are listed in Tab. 6. The RMSE 
of corresponding points pre- and post- adjustment is shown in Tab. 7. After mounting bias correction, that RMSE of corresponding points is great improved.

\begin{tabular}{|c|c|c|c|}
\hline \multirow{2}{*}{ Level-arm(meter) } & $X_{\text {offset }}$ & $Y_{\text {offset }}$ & $Z_{\text {offset }}$ \\
\cline { 2 - 4 } & $4.0768-\mathrm{e} 5$ & $5.41939-\mathrm{e} 4$ & $1.67461-\mathrm{e} 4$ \\
\hline \multirow{2}{*}{ Bore-sight(radian) } & $\alpha$ & $\beta$ & $\gamma$ \\
\cline { 2 - 4 } & $-5.13916-\mathrm{e} 3$ & $2.38587-\mathrm{e} 3$ & $-7.00623-\mathrm{e} 4$ \\
\hline
\end{tabular}

Tab. 6 Mounting parameters

\begin{tabular}{|l|l|l|l|}
\hline & $\mathrm{X}(\mathrm{m})$ & $\mathrm{Y}(\mathrm{m})$ & $\mathrm{Z}(\mathrm{m})$ \\
\hline Pre & 3.577878 & 3.752160 & 0.834701 \\
\hline Post & 0.149332 & 0.142443 & 0.036839 \\
\hline \multicolumn{3}{|c|}{ Tab. 7 RMSE of tie points } \\
\hline
\end{tabular}

We select several profiles in overlapping region to check the offsets among strips. Fig. 5a shows a certain profile of original LIDAR strips, there are obvious offsets between gable roof and ground. Fig. 5 shows the same profile of LIDAR strips after adjustment, roof and ground are coincided very well. To check the horizontal accuracy between strips, LIDAR points are rendered by intensity. Fig. 6 shows the overlay drawing of strip 1-4. Mounting bias causes significant horizontal displacement (Fig. 6a). After adjustment, the runway center line is accurately registered (Fig. 6b).

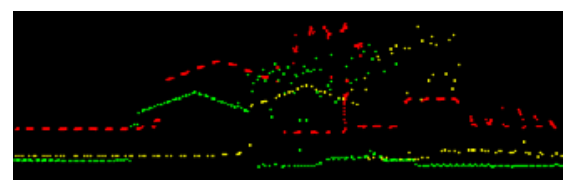

a. Before adjustment

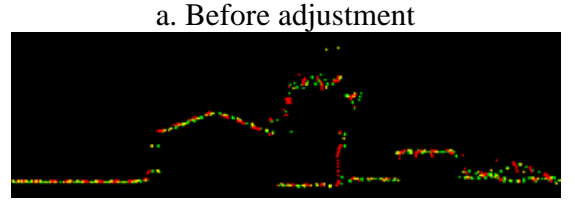

b. After adjustment

Fig. 5 Contrast of profile
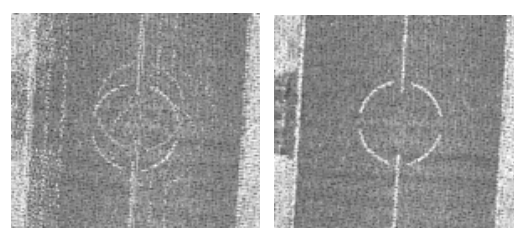

a. Before adjustment b. After adjustment

Fig. 6 Contrast of LIDAR Intensity

3.2.2 Experiment 2: In this experiment, mounting parameters acquired from experiment 1 are applied to corrected strips 5-8 directly. Elevation profiles of each strip pair $(5-6,6-7,7-8)$ are selected for visual inspection. The color of laser points are specified by strip number, color scheme is in Tab. 1 . As shown in Fig. 7, a large misalignment exists between raw strips. After correction by mounting parameters, the misalignment is almost eliminated, and the relative accuracy between strips is greatly improved.

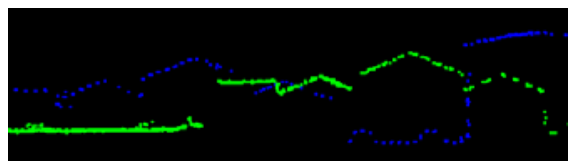

a. pre-corrected of strip $5 \sim 6$

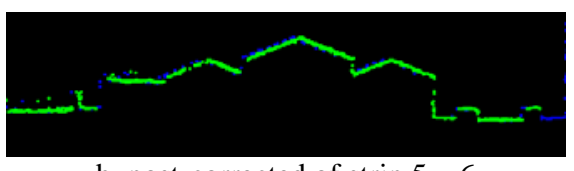

b. post-corrected of strip $5 \sim 6$

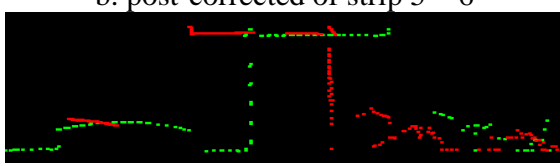

c. pre- corrected of strip $6 \sim 7$

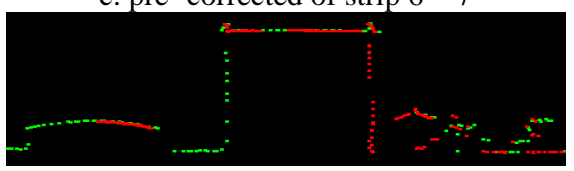

d. post- corrected of strip $6 \sim 7$

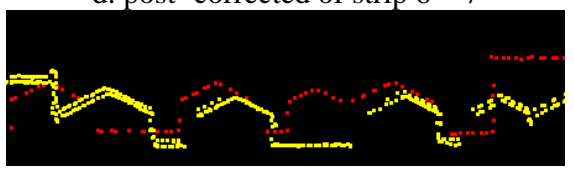

e. pre- corrected of strip $7 \sim 8$

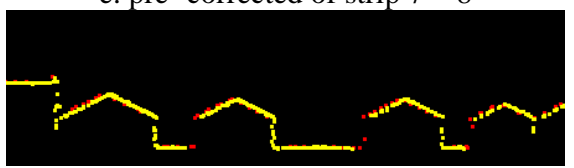

f. post- corrected of strip $7 \sim 8$

Fig. 7 Contrast of profiles

For rectified LIDAR data, differential DSM (D-DSM) is used to quantitatively analyze the relative accuracy between strips. Choose strip 6 and 7 as an example. Firstly, each strip is interpolated into regular DSM with resolution of 1 meter by triangle interpolation. Then hill shading of each DSM is created to enhance 3D effect. Finally, difference of overlapping region is calculated to form a D-DSM. To analyze the magnitude and distribution of residual error between strips, two strips DSM overlay with hillshading, and D-DSM are overlapped together. Hypsometric layer of D-DSM with interval of $0.2 \mathrm{~m}$ is generated. If the differences in the range of $-0.2 \sim 0.2 \mathrm{~m}$, the color is transparent. As shown in Fig. 8, the residual error between strips is small on the whole. Especially at ground region and building roofs, the residuals are less than $0.2 \mathrm{~m}$. But at vegetation region and surrounding buildings, the residuals are larger. For vegetation region, the collection of each strip is inconsistent, so larger residuals are inevitable. To investigate neighboring building area, three typical regions, identified by three red rectangles, are selected. Region a. includes high buildings and vegetation; region $b$. includes low buildings and high buildings and only low buildings exist in region c. Partial enlarged detail of these regions can been found in Fig. 8. Comparison of these regions, it can be found that: (1) residuals are distributed on both sides of buildings along scanning direction. (2) Residuals are bilateral symmetry on both sides of buildings. (3) High buildings have larger residuals scope than low buildings. Two profiles on building region are selected to inspect horizontal accuracy between strips, one profile is on a high building, and the other is on a low building. In Fig. 9, laser footprints in different strips are fitting well in both horizontal and vertical direction. As the presence of occlusion, obvious gaps exist on the side of buildings which away from scanning center line. Along scanning direction, gaps are bilateral symmetry on both sides of buildings. Comparing red circle between Fig. 9a. and Fig. 9b., higher buildings cause larger gaps. These gaps lead to erroneous interpolation results, and cause large residuals. Thus, the big residuals surrounding buildings are cause by occlusions. After correction by mounting parameters, relative accuracy of strips is greatly improved. 

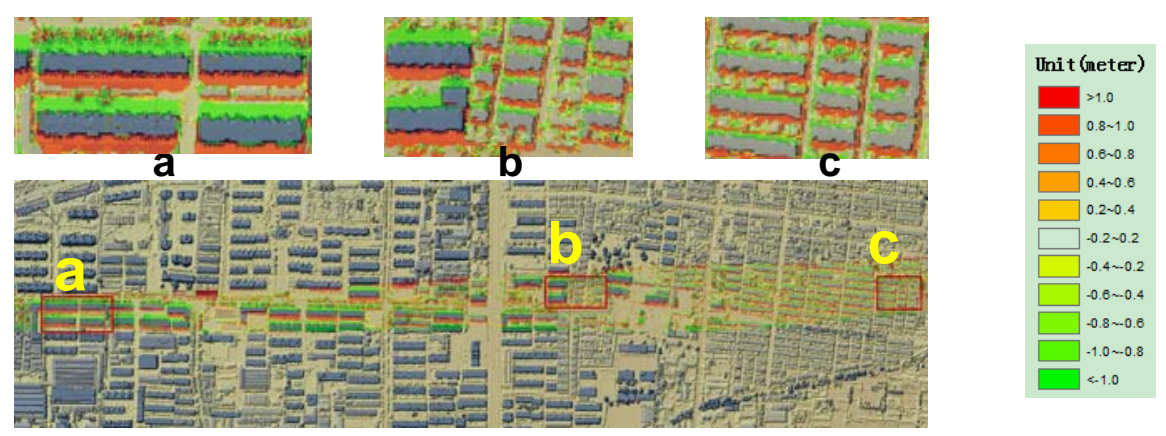

Fig. 8 Hypsometric layer map of overlapping region D-DSM

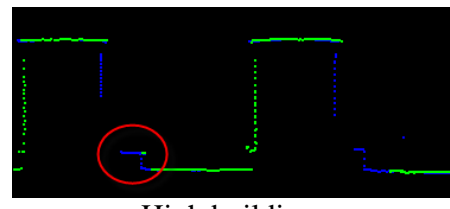

a. High buildings

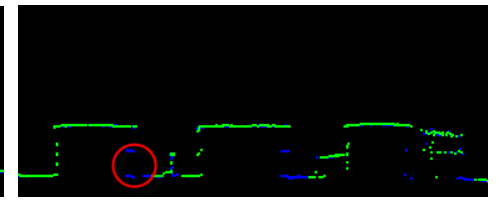

b. Low buildings

Fig. 9 Profiles of buildings

\section{CONCLUSIONS}

LIDAR system bias calibration can improve data accuracy effectively. It is very important for LIDAR applications. Prior methods exist more or less shortages in feasibility, accuracy and automaticity. This paper introduces an automated method for airborne LIDAR mounting biases calibration. The calibration process uses the LIDAR direct geo-referencing model to solve the level-arm offsets and bore-sighting angles. As LIDAR points are discrete sampling, real conjugated points don't exist between LIDAR strips; Virtual Corresponding Point Model (VCPM) is used as corresponding primitive. Using the given rules, virtual corresponding points easily associate with real laser footprints. Then the adjustment based on corresponding points is applied directly. Automatic flow is designed to determine corresponding points between LIDAR strips, which contains SIFT matching, RANSAC estimation and classification.

A set of real LIDAR strips is used to test proposed method. The experiment is divided into two steps. Firstly, 4 LIDAR strips are used to solve system mounting parameters with our method. Then the remainder 4 strips are corrected by acquired mounting parameters and the relative accuracy is analyzed to verify the efficiency of calibrated parameters. The results show the feasibility and efficiency of the proposed procedure.

\section{References}

Abedini, A., Hahn M., et al. An Investigation into The Registration of LIDAR Intensity Data And Aerial Images Using The SIFT Approach [C]. ISPRS08 (B1: $169 \mathrm{ff}), 2008$.

Baltsavias, E. P.. Airborne Laser Scanning: Basic Relations and Formulas [J]. ISPRS Journal of Photogrammetry and Remote Sensing, 1999, 54(2-3): 199-214.

Burman H. Calibration And Orientation of Airborne Image and Laser Scanner Data Using GPS and INS [D]. Stockholm: Royal Institute of Technology, 2000.

Crombaghs M. J E., Brügelmann R., DE MIN E. On The Adjustment Of Overlapping Strips of Laser Altimeter Height Data [J]. International Archives of Photogrammetry and Remote Sensing, 2000, 33(B3/1): 224-231.

Filin S., Vosselman G.. Adjustment of Airborne Laser Altimetry Strips [J]. International Archives of Photogrammetry, Remote Sensing and
Spatial Information Sciences, 2004, 34(B3): 285-289.

Fischler M. A., Bolles R. C. Random Sample Consensus: A Paradigm for Model Fitting with Applications to Image Analysis and Automated Cartography [J]. Communications of the ACM, 1981, 24(6): 381-395.

Habib A F, Al-Durgham M, Kersting A P, et al. Error Budget of LIDAR Systems and Quality Control of the Derived Point Cloud [C]. Proceedings of the ISPRS 2008 Congress. Beijing: ISPRS, 2008.

Habib, A., Bang K. I., et al. Alternative Methodologies For LIDAR System Calibration [J]. Remote Sensing. 2010, 2(3), 874-907.

Habib A F, Bang K I, Shin S W, et al. LIDAR System Self-calibration Using Planar Patches from Photogrammetric Data [R]. Padua: Fifth International Symposium on Mobile Mapping Technology (MMT'07), 2007.

Kilian J., Haala N., Englich M.. Capture And Evaluation of Airborne Laser Scanner Data [J]. International Archives of Photogrammetry and Remote Sensing, 1996, 31: 383-388.

Lowe, D G. Distinctive Image Features from Scale-Invariant Keypoints [J]. International Journal of Computer Vision. 2004. 60(2): 91-110.

Moré J. J. The Levenberg-Marquardt Algorithm: Implementation and Theory [M]. WATSON G A. Numerical Analysis: 603. New York: Springer, 1978: 105-116.

Morin K W. Calibration Of Airborne Laser Scanners [D]. Calgary: University of Calgary, 2002.

Schenk T. Modeling and Analyzing Systematic Errors in Airborne Laser Scanners [R]. Technical Notes in Photogrammetry No 19. Columbus: Ohio State University, 2001.

Shan J., Toth C. K.. Topographic Laser Ranging and Scanning: Principles and Processing [M]. Boca Raton: CRC Press, 2008.

Shepard M. K., Campbell B. A., et al. The Roughness of Natural Terrain: A Planetary and Remote Sensing Perspective [J]. Journal of Geophysical Research-Planets, 106(E12): 32777-95. 2001.

Skaloud J., Lichti D.. Rigorous Approach to Bore-sight Self-calibration in Airborne Laser Scanning [J]. ISPRS Journal of Photogrammetry and Remote Sensing, 2006, 61(1): 47-59.

Vosselman G., Maas H. G.. Adjustment And Filtering of Raw Laser Altimetry Data [C]. Proceedings OEEPE Workshop on Airborne Laser Scanning and Interferometric SAR for Detailed Digital Elevation Models. [S.l.]: OEEPE Publication, 2001.

Vosselman G, Mass H G. Airborne and Terrestrial Laser Scanning [M]. Dunbeath: Whittles Publishing, 2010.

Zhang Xiaohong. Precise Point Positioning Evaluation and Airborne LIDAR Calibration [R]. Copenhagen: Danish National Space Center, 2005. 